\title{
Efectividad de la Distracción Tibiofemoral en la Funcionalidad Adicionada al Tratamiento Convencional en Pacientes Mayores de 50 Años con Osteoartritis de Rodilla. Un Estudio Piloto
}

\author{
Effectiveness of Tibiofemoral Distraction in the Functionality Added to Conventional \\ Treatment in Patients over 50 Years with Knee Osteoarthritis. A Pilot Study
}

\author{
Angelo Rojas-Vidal ${ }^{1,3}$; Cecilia Valencia ${ }^{2}$ \& Luis A. Salazar ${ }^{3}$
}

ROJAS-VIDAL, A.; VALENCIA, C. \& SALAZAR, L.A. Efectividad de la distracción tibiofemoral en la funcionalidad adicionada al tratamiento convencional en pacientes mayores de 50 años con osteoartritis de rodilla. Un estudio piloto. Int. J. Morphol., 36(1):267$272,2018$.

RESUMEN: Osteoartritis (OA) es una de las enfermedades reumáticas más frecuentes y la más común enfermedad articular, afecta principalmente al cartílago articular y el hueso subcondral de una articulación sinovial resultando en la incapacidad articular. La OA es reconocida como una causa substancial de discapacidad, con significativos costos sociales y financieros debidos a las frecuentes intervenciones médicas y quirúrgicas además del ausentismo laboral. El objetivo del estudio fue determinar si la distracción tibiofemoral adicionada al tratamiento convencional en pacientes con osteoartritis de rodilla grado 3, presenta mejoras en el dolor, rango de movimiento y funcionalidad de la extremidad inferior, en comparación al tratamiento convencional propuesto por el MINSAL por sí solo. Esta investigación corresponde a un estudio piloto, con diseño experimental del tipo ensayo clínico aleatorizado (ECA), controlado, ciego simple y con una muestra equilibrada. La muestra corresponde a pacientes reclutados del Hospital San Borja Arriarán (Santiago, Chile) con diagnóstico médico de OA de rodilla grado 3. Se realizaron dos mediciones, una al inicio de las diez sesiones y otra al final del tratamiento, donde se midió el dolor con la escala visual análoga (EVA), el rango de movimiento articular pasivo con goniometría y la capacidad funcional con el test de marcha de 6 minutos y el cuestionario de WOMAC. Al comparar ambos grupos muestran diferencias estadísticamente significativas en la funcionalidad, el dolor y distancia de marcha durante 6 minutos. La distracción tibiofemoral adicionada al tratamiento convencional presentó mejoras clínicas significativas cuando comparada con la aplicación del tratamiento convencional de $\mathrm{OA}$ de rodilla.

PALABRAS CLAVE: Osteoartritis de rodilla; Distracción tibiofemoral; Tracción.

\section{INTRODUCCIÓN}

La Osteoartritis (OA) es definida como un grupo heterogéneo de condiciones que conducen a síntomas y signos articulares, asociados a defectos del cartílago articular, además de cambios en el hueso subcondral y en los márgenes articulares (Altman et al., 1986). Incluyendo ligamentos, cápsula articular, membrana sinovial y músculos peri articulares. De esto se desprende que la OA es un proceso de daño articular con diferentes etiologías, pero con un patrón de presentación similar, tanto biológico, morfológico y clínico (Radrigán, 2004).

Mundialmente se ha reportado incidencias entre 164 y 240 casos por cada 100.000 habitantes para OA de rodilla (Kallman et al., 1990; Wilson et al., 1990). En Chile, de acuerdo a los datos de la encuesta nacional de salud (ENS) del 2009, un 3,8\% de la población adulta refiere padecer de OA, siendo más frecuente en mujeres y mayor en la medida que aumenta la edad. En mayores de 60 años se estima que más del $80 \%$ presenta alteraciones radiológicas de $\mathrm{OA}$ en al menos una articulación.

La importancia de la OA radica en su alta frecuencia, la presencia de molestias e importantes limitaciones en actividades diarias y una evidente restricción en la participación social y la subsecuente disminución en calidad de vida. Actualmente, el tratamiento de la OA está enfocado al manejo de los síntomas, y no a evitar la progresión de la enfermedad (Hinman et al., 2003; Pollard et al., 2008). Las

${ }^{1}$ Servicio Kinesiología del Hospital Clínico San Borja Arriarán (HCSBA), Santiago; Chile.

${ }^{2}$ Instituto de Investigación Materno Infantil (IDIMI), Facultad de Medicina, Universidad de Chile, Santiago, Chile.

${ }^{3}$ Centro de Biología Molecular y Farmacogenética, Departamento de Ciencias Básicas, Facultad de Medicina, Universidad de La Frontera, Temuco, Chile. 
estrategias utilizadas van desde el tratamiento farmacológico, no farmacológico, hasta intervenciones quirúrgicas, dependiendo del grado de OA que posea el paciente (Deyle et al., 2000). Dentro del tratamiento no farmacológico se encuentra la rehabilitación basada en kinesiología, que busca manejar y/o atenuar los deterioros estructurales y funcionales causados por la OA; a través de la movilización y modulación del dolor e intervención de la funcionalidad del paciente (giros en cama, transición al sedente, transición de sedente a bípedo, marcha). El tratamiento kinésico habitual incluye técnicas de fisioterapia, ejercicio físico, además de fortalecimiento muscular (Pisters et al., 2007). Sin embargo, hay otros procedimientos kinésicos utilizados con objetivos específicos, como es la terapia manual. Definida como la entrega o aplicación de fuerzas manuales a través de procedimientos destinados a modificar la calidad y el rango de movimiento de la articulación diana y los tejidos blandos circundantes (Abbott et al., 2009; Pinto et al., 2011). La distracción tibiofemoral es una técnica de terapia manual, entendida como la separación de dos superficies articulares perpendiculares al plano de la articulación o de tratamiento, esta técnica es ampliamente utilizada en la clínica para el tratamiento de OA; sin embargo, a la fecha no se reportan estudios que evalúen los efectos clínicos de la distracción tibiofemoral en OA de rodilla.

El objetivo principal de este estudio piloto es determinar la efectividad de la técnica de distracción tibiofemoral en la funcionalidad, disminución del dolor, grado de rigidez y rangos articulares de la miembro inferior en pacientes con OA de rodilla grado 3 .

\section{MATERIAL Y MÉTODO}

Participantes: Este estudio fue realizado en un grupo de pacientes diagnosticados con OA de rodilla grado 3, atendidos en el Servicio de Kinesiología del Hospital Clínico San Borja Arriarán (HCSBA), Santiago, Región Metropolitana, Chile. Previo a la selección de los pacientes, fue necesario la firma de un consentimiento informado, el que fue aprobado junto con la investigación a evaluación por parte del Comité Ético Científico (CEC) del Servicio de Salud Metropolitano Central (SSMC).

Los pacientes que participaron en esta investigación se acogieron a los siguientes criterios de inclusión: Tener diagnostico radiológico de osteoartritis grado 3 según criterios de clasificación de Kellgren \& Lawrence (Pinto et al.), presentar signos clínicos de rigidez matinal menor a $30 \mathrm{mi}-$ nutos, crepitaciones y sensibilidad ósea (dolor a la palpación de los márgenes articulares), engrosamiento óseo de la rodilla, edad superior a 50 años y firma de consentimiento informado. Los criterios de exclusión, fueron presentar lesiones de rodilla, sufrir de otras condiciones como artroplastías del miembro inferior, condiciones que requieran resolución quirúrgica, artritis séptica o por cristales, haber estado en tratamiento con infiltración de inmunosupresor en los últimos seis meses, presencia de intervención quirúrgica de rodilla, aumento de temperatura local al tacto, problemas cardiacos o respiratorios que impidan la realización del test de marcha, déficits cognitivos o barreras lingüísticas que dificulten la capacidad de leer, entender, o firmar el formulario de consentimiento, el test de WOMAC o la escala visual análoga.

Distribución de la muestra: La muestra total fue de 20 pacientes, esto se obtuvo a través de una aleatorización equilibrada en donde cada paciente pudo elegir entre 20 sobres negros sellados, en ellos se escribió una "X" y una "O", en donde $\mathrm{X}$ corresponde al grupo experimental y $\mathrm{O}$ al grupo control. La muestra fue dividida en 2 grupos, cada uno de 10 pacientes. Después de la asignación al azar, los pacientes fueron registrados en una ficha con datos clínicos personales, realizando una evaluación previa a la intervención y una en la décima sesión, finalmente se realizó seguimiento de los pacientes el cual se detalla.

Intervención: Previo al inicio de sesiones, se realizó un registro de datos personales y medición de la funcionalidad, dolor, rango articular y test de marcha de 6 minutos. Al finalizar las sesiones se volvió a evaluar y medir estos parámetros. Al grupo control solo se le realizó el tratamiento convencional propuesto por el MINSAL, que incluye la aplicación de compresa húmedo caliente por 10 minutos, el uso de bicicleta estática por 10 minutos, finalizando con el fortalecimiento muscular de las EEII por 10 minutos. El tratamiento se efectuó 3 veces por semana con una duración de 10 sesiones en total. Al grupo experimental, se le realizó el tratamiento convencional propuesto por el MINSAL e incluyó la distracción tibiofemoral, dentro de un tiempo total por sesión de $40 \mathrm{mi}$ nutos, brevemente, en los 10 minutos iniciales se aplicó compresa húmedo caliente para preparar los tejidos, posteriormente se aplicó la técnica de distracción tibiofemoral durante un periodo de 5 minutos, seguido de 10 minutos de bicicleta estática y 10 minutos de ejercicio de fortalecimiento de la musculatura en miembro es inferiores (EEII), finalizando con 5 minutos de aplicación de distracción tibiofemoral.

Variables: La funcionalidad se midió con el cuestionario de OA de las Universidades de Western Ontario y McMaster (WOMAC) (Bellamy et al., 1988), el que fue diseñado para medir dolor, rigidez y capacidad funcional a nivel de cadera y rodilla, que presentan osteoartritis. Consta de 24 ítems actividades de la vida diaria que exploran tres dimensiones: 
dolor (5 ítems), rigidez ( 2 ítems) y grado de dificultad con ciertas actividades físicas (17 ítems). Recibiendo una puntuación de 0 a 4 puntos $(0=$ ninguno; $1=$ poco; $2=$ bastante; 3= mucho; 4= muchísimo). La puntuación global se calcula sumando las puntuaciones de las 3 sub-escalas, siendo el puntaje máximo total de 96 puntos. El valor más cercano a cero indica un mejor nivel funcional logrado. La escala visual análoga (EVA) se utilizó para medir los niveles de dolor, se presenta como una línea de $10 \mathrm{~cm}$, anclado por descriptores verbales, por lo general "no dolor" y "el peor dolor imaginable". Se solicita al paciente marque una línea de $100 \mathrm{~mm}$ para indicar la intensidad del dolor. La puntuación se mide desde el anclaje de cero a la marca del paciente, utilizando una regla milimétrica para medir la puntuación del paciente (Williamson \& Hoggart, 2005). El rango articular se evaluó con goniometría, mediante el uso de un instrumento formado por dos brazos y un cuerpo en el que se sitúa un transportador de ángulos que permite obtener la medida (Gil Fernández \& Zuil Escobar, 2012). Finalmente, la distancia recorrida se evalúa con un test de marcha de 6 minutos, que es una prueba funcional cardiorrespiratoria, consistente en medir la distancia máxima que puede recorrer un sujeto durante 6 minutos (Guyatt et al., 1985; Gutiérrez-Clavería et al., 2009).

Análisis estadístico: Se evaluó cuatro variables dependientes, la funcionalidad con el cuestionario WOMAC, el dolor con la EVA, rango articular pasivo con goniometría y distancia recorrida con el test de marcha de 6 minutos. Dado que los datos obtenidos de la muestra no tienen una distribución normal, se realizó un análisis estadístico mediante prueba de muestras independientes y prueba t pareada para evaluar si existe o no diferencias estadísticamente significativas. Se consideró para todos los análisis, un error alpha $=0,05$.

\section{RESULTADOS Y DISCUSIÓN}

La Figura 1 muestra el flujo de pacientes y de trabajo. Obteniendo una muestra equilibrada de veinte pacientes, luego se realizó la aleatorización y designación de grupos control y experimental. En el seguimiento del grupo control, un paciente interrumpió la intervención por problemas familiares, por lo que los grupos quedaron conformados por 9 controles y 10 experimentales. Al finalizar el estudio se analizó los resultados con el principio de "intención de tratar". La Tabla I resume las características basales de los pacientes estudiados. No se encontraron diferencias significativas al comparar las diferentes variables analizadas (promedio de edad, distribución por género, índice de masa corporal, porcentaje del miembro afectada (derecha-izquierda) y duración de los síntomas).
Para confirmar la efectividad de la aplicación de la técnica de distracción tibiofemoral adicionada al tratamiento convencional en la suma de las puntuaciones de función, dolor y rigidez de la miembro inferior, se realizó el cuestionario de WOMAC, en el que se obtuvo una disminución significativa del puntaje obtenido por el grupo experimental en comparación al grupo control, $(\mathrm{p}=0,004)$; demostrando que la aplicación de distracción tibiofemoral es efectiva para los diversos parámetros medidos por este test. Los valores obtenidos son resumidos en la Tabla II.

La reducción en el puntaje de WOMAC obtenida por el grupo experimental fue de $22 \%$, este porcentaje excede el nivel de $20 \%$ a $25 \%$ sugerido como mínimamente significativos por Barr et al. (1994), siendo notoriamente mejores los puntajes para el grupo experimental que los observados en el grupo control que fue de un $16 \%$ de disminución. Este resultado se asemeja al obtenido por Deyle et al. (2000), quienes reportaron una disminución del puntaje WOMAC de un 55,8\% sobre los niveles basales en el grupo de tratamiento al utilizar "terapia manual" versus un grupo placebo consistente en ultrasonido a una intensidad sub-terapéutica.

En el año 2005 este mismo grupo de investigación reporta que su grupo de "tratamiento clínico", consistente en la utilización de terapia manual, bicicleta estacionaria y supervisión de ejercicios por un terapeuta físico, mostró una disminución en el puntaje WOMAC superior al grupo que realizó ejercicios sin supervisión en el hogar (Deyle et al., 2005). Finalmente, Rhon et al. (2013) muestran la efectividad de adicionar ejercicios de perturbación a la "terapia manual" obteniendo una disminución del puntaje WOMAC de $53 \%$ al mes de tratamiento, $51 \%$ a los tres meses, porcentaje mantenido a los seis meses.

Otro test aplicado para evaluar funcionalidad del miembro afectada por OA de rodilla grado 3 fue el test de marcha de 6 minutos. En el presente estudio, se obtuvo un aumento de 13 metros en promedio de distancia recorrida durante este periodo de tiempo, que representa un $14 \%$ de aumento para el grupo experimental (Tabla II). Estos resultados concuerdan con los obtenidos por Deyle et al. (2000) que reportan un aumento en el test de marcha de 6 minutos de un 13,1\%, así como un aumento de $10 \%$ (Deyle et al., 2005).

Al evaluar el nivel de dolor con EVA, se observó también una diferencia estadísticamente significativa entre los grupos $(\mathrm{p}=0,007)$; con una disminución del nivel de dolor de 3 puntos en el grupo control. Este resultado reafirma el parámetro medido en el cuestionario WOMAC; sin embargo, el test de EVA no ha sido utilizado anteriormente en $\mathrm{OA}$ de rodilla. 


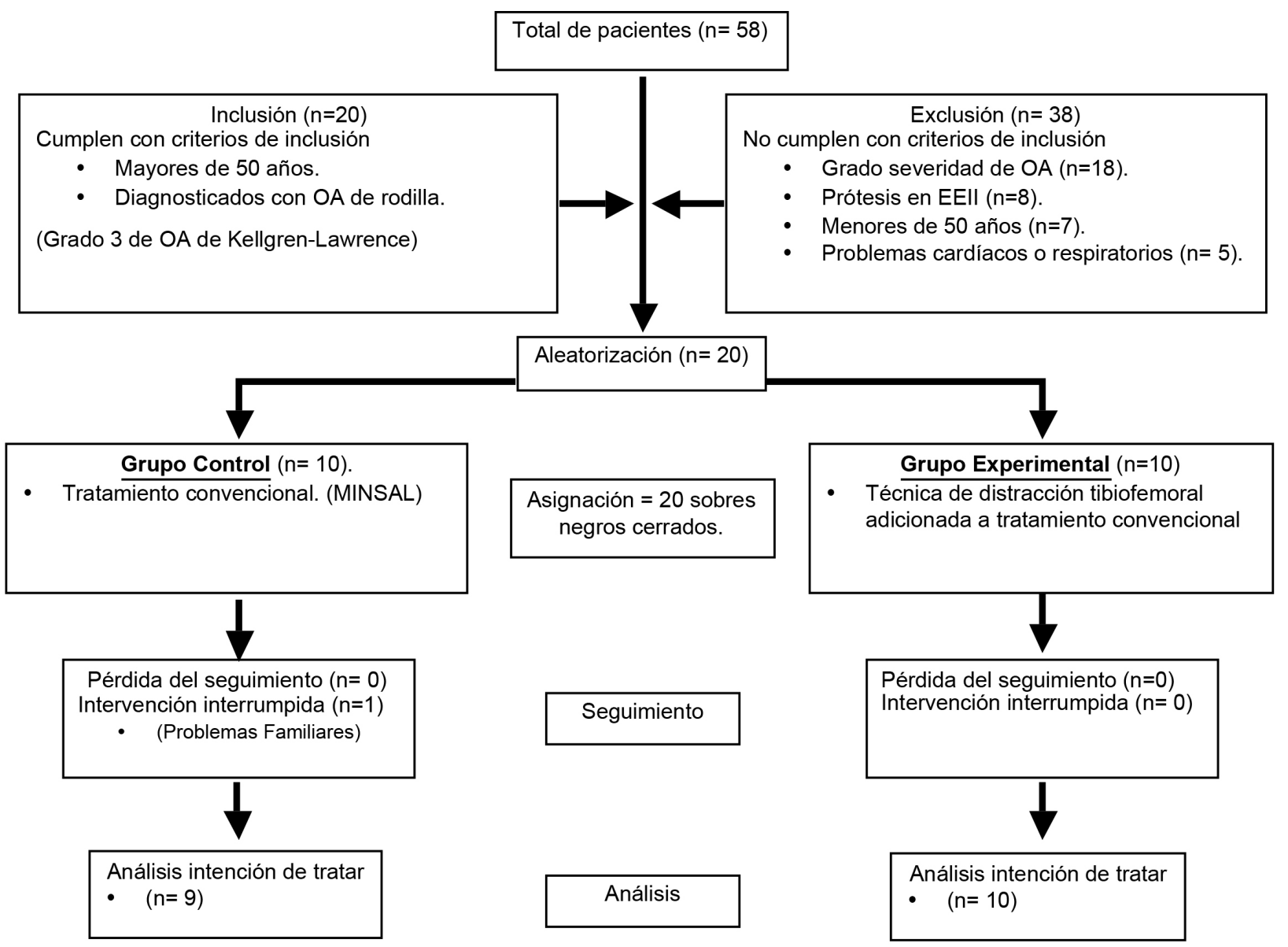

Fig. 1. Flujograma de pacientes.

En relación al rango articular de flexión de rodilla se obtuvo un aumento significativo del grupo control de $18.3^{\circ}$ en comparación al grupo experimental de solo $3^{\circ}(\mathrm{p}=0,007)$. Para el rango articular de extensión de rodilla no hubo cambio estadísticamente significativo ni concluyente; este resultado es explicado por la condición basal de ambos grupos, ya que ambos presentaban rango articular completo de extensión de rodilla, en la evaluación inicial, excepto en dos pacientes del grupo control. Las mediciones de rango articular realizadas con goniometría solo son analizadas en la presente investigación, ya que en la investigación de (Deyle et al., 2005) son utilizadas como criterio de selección del tipo de terapia manual a utilizar.

Cabe señalar que los estudios anteriormente mencionados no especifican el grado de OA de los pacientes diagnosticados con esta patología, así como no especifican la técnica utilizada como "terapia manual", aplicando distintas técnicas de terapia manual a los integrantes de un mismo grupo experimental y debido a que los criterios de inclusión
Tabla I. Características clínicas y demográficas basales de los grupos estudiados.

\begin{tabular}{lcc}
\hline Características & $\begin{array}{c}\text { Control } \\
(10)\end{array}$ & $\begin{array}{c}\text { Experimental } \\
(10)\end{array}$ \\
\hline Edad (años) & $62,2 \pm 8,49$ & $60,8 \pm 7,40$ \\
Sexo & & \\
$\quad$ Femenino & $80 \%$ & $100 \%$ \\
$\quad$ Masculino & $20 \%$ & $0 \%$ \\
Índice de masa corporal & $30,89 \pm 5,01$ & $29,60 \pm 3,66$ \\
Miembro afectado & & \\
$\quad$ Derecha & $70 \%$ & $50 \%$ \\
$\quad$ Izquierda & $30 \%$ & $50 \%$ \\
Duración de los síntomas & $5,7 \pm 3,71$ & $5,6 \pm 2,63$ \\
Puntuación total WOMAC & $52 \pm 19,1$ & $48 \pm 14,2$ \\
Test de marcha 6 min. & $264 \pm 122,2$ & $226 \pm 79,9$ \\
Dolor (EVA) & $8 \pm 1,3$ & $8,3 \pm 1,5$ \\
ROM (grados) & & \\
$\quad$ Flexión & $94,78 \pm$ & $87,5 \pm 20,31$ \\
$\quad$ Extensión (negativo) & $3,7 \pm 4,32$ & $1,8 \pm 3,82$ \\
\hline
\end{tabular}


Tabla II. Comparación entre grupo control y experimental.

\begin{tabular}{|c|c|c|c|c|c|c|c|c|}
\hline \multicolumn{5}{|c|}{ Grupo Control } & \multicolumn{4}{|c|}{ Grupo Experimental } \\
\hline Variables & $\begin{array}{c}\text { Basal } \\
(10)\end{array}$ & $\begin{array}{c}\text { Final } \\
(9)\end{array}$ & Diferencia & Valor $\mathrm{p}$ & $\begin{array}{c}\text { Basal } \\
(10)\end{array}$ & $\begin{array}{c}\text { Final } \\
(10)\end{array}$ & Diferencia & Valor $\mathrm{p}$ \\
\hline WOMAC & 56 & 47 & 9 & 0,452 & 49 & 38 & 11 & 0,004 \\
\hline Dolor (EVA) & 8 & 7 & 1 & 0,257 & 8 & 5 & 3 & 0,007 \\
\hline Rango articular flexión & 94,78 & 97,78 & 3 & 0,472 & 87,50 & 105,80 & 18,3 & 0,001 \\
\hline Rango articular extensión & $-4,11$ & $-3,44$ & 0.67 & 0,686 & $-1,80$ & 0,00 & 1,8 & 0,317 \\
\hline Test de marcha 6 minutos & 264 & 277 & 13 & 0,374 & 226 & 258 & 32 & 0,008 \\
\hline
\end{tabular}

Medias e intervalos de confianza del $95 \%$ para el índice de osteoartritis de Western Ontario \& McMaster Universities (WOMAC), escala visual análoga (EVA) de dolor, test de marcha de 6 minutos y rangos articulares de extensión y flexión de rodilla.

son más amplios presentan un mayor tamaño muestral en comparación a este trabajo. Otro aspecto importante considerar, en el escenario puntual de estudio piloto, es la significancia estadística, esta puede no resolver la incertidumbre clínica, dado que es un concepto exclusivamente matemático y no de garantía de calidad, ya que depende de dos elementos esenciales: la magnitud de la diferencia que queremos probar y el tamaño de la muestra, reduciendo así la variabilidad secundaria al azar. Para futuras investigaciones se recomienda aumentar el número de pacientes a tratar (tamaño muestral), aumentar la cantidad de sesiones, realizar evaluaciones a mediano y largo plazo sobre la funcionalidad, realizar una técnica de terapia manual en el grupo control que simule la distracción tibiofemoral, para evitar el efecto placebo de la misma.

La aplicación de la técnica de distracción tibiofemoral posee ciertas ventajas sobre otras técnicas, como la terapia interferencial, ultrasonido, onda corta o baños de parafina, dado el bajo costo, fácil implementación, requiriendo de un terapeuta especializado. Dado que la OA de rodilla en nuestro país posee un alta prevalencia, es necesario complementar las terapias actuales principalmente con aquellas que presenten un menor costo para su aplicación, pero una mayor efectividad en el control de la sintomatología en pacientes con esta patología.

ROJAS-VIDAL, A.; VALENCIA, C. \& SALAZAR, L.A. Effectiveness of tibiofemoral distraction in the functionality added to conventional treatment in patients over 50 years with knee osteoarthritis. A pilot study. Int. J. Morphol., 36(1):267-272, 2018.

SUMMARY: Osteoarthritis (OA) is one of the most frequent rheumatic diseases and the most common joint disease, primarily affecting joint cartilage and the subchondral bone of a synovial joint resulting in the $\mathrm{y}$ articular dysfunction. OA is recognized as a cause of disability, with significant social and financial costs due to medical, surgical interventions in addition to frequent absenteeism at work. The objective was to determine if tibiofemoral distraction, in conjunction with conventional therapy in patients with knee osteoarthritis grade 3 , improved performance of the lower extremity in comparison to the conventional treatment alone, as proposed by the Ministry of Health (MINSAL). This research is a pilot, prospective randomized clinical trial (RCT), controlled, single-blind and with a balanced sample. The sample was recruited from patients of the Hospital San Borja Arriarán (Santiago, Chile) with medical diagnosis of knee OA grade 3. Two measurements were performed, one at the beginning of the 10 sessions and at the end of treatment, which was measured with the functionality questionnaire of $\mathrm{OA}$ of the Western Ontario and McMaster Universities (WOMAC), the pain visual analog scale (VAS), the passive joint range of motion with goniometry, and distance to the Test 6-minute walk. When comparing the two groups showed statistically significant differences in functionality, pain, distance walked and joint range, except in knee grade extension. The tibiofemoral distraction added to conventional treatment functional improvement in patients with knee OA grade 3.

KEY WORDS: Knee osteoarthritis; Tibiofemoral distraction; Traction.

\section{REFERENCIAS BIBLIOGRÁFICAS}

Abbott, J. H.; Robertson, M. C.; McKenzie, J. E.; Baxter, G. D.; Theis, J. C.; Campbell, A. J. \& MOA Trial team. Exercise therapy, manual therapy, or both, for osteoarthritis of the hip or knee: a factorial randomised controlled trial protocol. Trials, 10:11, 2009.

Altman, R.; Asch, E.; Bloch, D.; Bole, G.; Borenstein, D.; Brandt, K.; Christy, W.; Cooke, T. D.; Greenwald, R. \& Hochberg, M. Development of criteria for the classification and reporting of osteoarthritis. Classification of osteoarthritis of the knee. Diagnostic and Therapeutic Criteria Committee of the American Rheumatism Association. Arthritis Rheum., 29(8):1039-49, 1986.

Barr, S.; Bellamy, N.; Buchanan, W. W.; Chalmers, A.; Ford, P. M.; Kean, W. F.; Kraag, G. R.; Gerecz-Simon, E. \& Campbell, J. A comparative study of signal versus aggregate methods of outcome measurement based on the WOMAC Osteoarthritis Index. Western Ontario and McMaster Universities Osteoarthritis Index. J. Rheumatol., 21(11):2106-12, 1994.

Bellamy, N.; Buchanan, W. W.; Goldsmith, C. H.; Campbell, J. \& Stitt, L. W. Validation study of WOMAC: a health status instrument for measuring clinically important patient relevant outcomes to antirheumatic drug therapy in patients with osteoarthritis of the hip or 
knee, J. Rheumatol., 15(12):1833-40, 1988

Deyle, G. D.; Henderson, N. E.; Matekel, R. L.; Ryder, M. G.; Garber, M. B. \& Allison, S. C. Effectiveness of manual physical therapy and exercise in osteoarthritis of the knee. A randomized, controlled trial. Ann. Intern. Med., 132(3):173-81, 2000.

Deyle, G. D.; Allison S. C.; Matekel R. L.; Ryder M. G.; Stang J. M.; Gohdes D. D.; Hutton J. P.; Henderson N. E., Garber M. B. Physical therapy treatment effectiveness for osteoarthritis of the knee: a randomized comparison of supervised clinical exercise and manual therapy procedures versus a home exercise program. Phys. Ther. (85): 1301-17, 2005.

Gil Fernández, M. \& Zuil Escobar, J. C. Fiabilidad y correlación en la evaluación de la movilidad de rodilla mediante goniómetro e inclinómetro. Fisioterapia, 34:73-8, 2012.

Gutiérrez-Clavería, M.; Beroíza, W. T.; Cartagena, S. C.; Caviedes, S. I.; Céspedes, G. J.; Gutiérrez-Navas, M.; Oyarzún, G. M.; Palacios, M. S. \& Schönffeldt, G. P. Prueba de caminata de seis minutos. Rev. Chil. Enferm. Respir., 25(1):15-24, 2009.

Guyatt, G. H.; Sullivan, M. J.; Thompson, P. J.; Fallen, E. L.; Pugsley, S. O.; Taylor, D. W. \& Berman, L. B. The 6-minute walk: a new measure of exercise capacity in patients with chronic heart failure. Can. Med. Assoc. J., 132(8):919-23, 1985.

Hinman, R. S.; Crossley, K. M.; McConnell, J. \& Bennell, K. L. Efficacy of knee tape in the management of osteoarthritis of the knee: blinded randomised controlled trial. BMJ, 327(7404):135, 2003.

Kallman, D. A.; Wigley, F. M.; Scott, W. W. Jr.; Hochberg, M. C. \& Tobin, J. D. The longitudinal course of hand osteoarthritis in a male population. Arthritis Rheum., 33(9):1323-32, 1990.

Pinto, D.; Robertson, M. C.; Hansen, P.; Abbott, J. H. \& MOA Trial Team. Economic evaluation within a factorial-design randomised controlled trial of exercise, manual therapy, or both interventions for osteoarthritis of the hip or knee: study protocol. BMJ Open, 1(1):e000136, 2011.

Pisters, M. F.; Veenhof, C.; van Meeteren, N. L.; Ostelo, R. W.; de Bakker, D. H.; Schellevis, F. G. \& Dekker, J. Long-term effectiveness of exercise therapy in patients with osteoarthritis of the hip or knee: a systematic review. Arthritis Rheum., 57(7):1245-53, 2007.

Pollard, H.; Ward, G.; Hoskins, W. \& Hardy, K. The effect of a manual therapy knee protocol on osteoarthritic knee pain: a randomised controlled trial. J. Can. Chiropr. Assoc., 52(4):229-42, 2008.

Radrigán, F. Tratamiento médico de la artrosis (osteoartritis) de rodilla en el anciano. Rev. Chil. Reumatol., 20(2):73-80, 2004.

Rhon, D.; Deyle, G.; Gill, N. \& Rendeiro, D. Manual physical therapy and perturbation exercises in knee osteoarthritis. J. Man. Manip. Ther, 21(4):220-8, 2013.

Williamson, A. \& Hoggart, B. Pain: a review of three commonly used pain rating scales. J. Clin. Nurs., 14(7):798-804, 2005.

Wilson, M. G.; Michet, C. J. Jr.; Ilstrup, D. M. \& Melton, L. J. 3rd. Idiopathic symptomatic osteoarthritis of the hip and knee: a population-based incidence study. Mayo Clin. Proc., 65(9):1214-21, 1990.

\author{
Dirección para correspondencia: \\ Prof. Dr. Luis Salazar Navarrete \\ Departamento de Ciencias Básicas \\ Facultad de Medicina \\ Universidad de La Frontera \\ Temuco \\ CHILE
}

E-mail: luis.salazar@ufrontera.cl

Recibido : 03-11-2017

Aceptado :03-01-2017 